\title{
El catálogo de los pretendientes de Penélope (Apollod., Ep. VII 26-30): tradición literaria y creación erudita*
}

\author{
Stefano Acerbo \\ Università di Pavia \\ acerboste@gmail.com \\ ORCID iD: https://orcid.org/0000-0003-0734-0190
The Catalogue of the Suitors of Penelope (Apollod., Ep. VII 26-30): Literary Tradition and Erudite Creation

El catálogo de los pretendientes de Penélope transmitido por Apolodoro presenta un caso interesante para estudiar el origen y el funcionamiento de las listas de nombres en la tradición erudita y mitográfica griega. Apolodoro menciona 129 pretendientes, frente a los 14 que aparecen nombrados en la Odisea, un hecho que, unido a la poca consistencia mitológica de estos personajes fuera del poema homérico, parece relegar el catálogo al campo de la simple invención libre, más que a la verdadera tradición mítica. Sin embargo, el estudio de los nombres de la lista y la comparación con otros catálogos, en parte transmitidos por papiros, permite reconocer algunos de los criterios utilizados por el compilador para construir la lista, como la interpretación del propio texto de Homero, y algunas estrategias compositivas que se pueden comparar con la práctica del centón. En este sentido, podemos apreciar cómo, también en este caso especial, la creación erudita tiene una relación muy fuerte con la tradición.

Palabras clave: Catálogos; pretendientes; centón; Odisea; Apolodoro el mitógrafo; erudición homérica.
The catalogue of Penelope's suitors transmitted by Apollodorus is an interesting case for studying the origin and functioning of the lists of names in the scholarly and mythological tradition. Apollodorus mentions 129 names, whereas the Odyssey only names 14 suitors, a fact that, together with the little mythological consistency of these characters outside the Homeric poem, seems to relegate this catalogue to the field of simple creation, rather than real tradition. However, the study of the names of the list and the comparison with other catalogues, some of them transmitted on papyri, allows us to recognize some of the criteria used to compile the list, such as the interpretation of Homer's text itself, and some further compositional strategies that can be compared with the practice of the cento. In this sense, we can appreciate that, also in this very specific case, the erudite creation has a strong connection with tradition.

Key words: Catalogues; the suitors of Penelope; cento; Odyssey; Apollodorus the mythographer; Homeric scholarship.

* Este artículo ha sido realizado en el contexto del proyecto de $\mathrm{I}+\mathrm{D}+\mathrm{i}$ «Estudios sobre tradición, transmisión y recepción de la mitografía griega. Antigüedad, Medievo y Humanismo» (PID2019-108931GBI00) bajo la dirección de la profesora Minerva Alganza Roldán y financiado por el Ministerio de Ciencia e Innovación. Agradezco a Irene Pajón Leyra su ayuda en la discusión sobre el papiro P.Oxy. LIII 3702. 
Cómo citar este artículo / Citation: Acerbo, Stefano (2021): «El catálogo de los pretendientes de Penélope (Apollod., Ep. VII 26-30): tradición literaria y creación erudita», Emerita 89 (2), pp. 227-249.

\section{Introducción}

Por lo general, la Biblioteca de Apolodoro el mitógrafo ${ }^{1}$ se considera una especie de repertorio donde se pueden encontrar, de una forma sencilla pero bastante completa, los relatos míticos más importantes de la Grecia antigua. Aunque esta visión no sea del todo errada, sin embargo, es parcial y no reconoce algunas de sus características más importantes y, al mismo tiempo, más difíciles de explicar. La Biblioteca, más que una recopilación de relatos, parece ser más bien un extraordinario repertorio de nombres. La mayor parte de ellos son simples eslabones que se sitúan en la cadena genealógica que estructura la obra de una forma continua. Esta estructura diferencia la Biblioteca del resto de la producción mitográfica de la edad imperial que conocemos y, a su vez, la acerca más bien a la mitografía de los siglos V y IV a. C., o incluso a un poema de época arcaica como el Catálogo de las mujeres atribuido a Hesíodo². Sin embargo, las estructuras genealógicas conviven con otra forma que permite a Apolodoro organizar los nombres, es decir, los catálogos. El estudio de esta forma de compilación mitográfica ofrece un material privilegiado para investigar el difícil equilibrio entre tradición y creación en la producción erudita.

${ }^{1}$ Según el ejemplo de Fowler 2013, p. 384, he decidido no utilizar el nombre de PseudoApolodoro. Aunque el autor de la Biblioteca no pueda ser Apolodoro de Atenas, nada en el texto lleva a suponer un intento de imitar al erudito ateniense, como sucede con mucha otra producción que denominamos con el término «pseudo». A nuestro parecer, para distinguir la Biblioteca de esta producción, es preferible evitar la expresión pseudo-Apolodoro, sobre todo cuando, como en este artículo, la ausencia de referencias a Apolodoro de Atenas excluye la posibilidad de confundir a los dos autores.

${ }^{2}$ Las otras obras de mitografía de edad imperial presentan una forma discontinua, con una división en secciones. Incluso cuando es posible reconocer un orden en la organización de la materia, las diferentes entradas siguen estando separadas las unas de las otras. Por tal razón se ha hablado de colecciones de anécdotas míticas (Goldhill 2009, p. 108). La estructura de la Biblioteca, en cambio, no permite una división en unidades autónomas (Yun Lee Too 2010, pp. 130-131). Sobre la semejanza entre Apolodoro y la mitografía del siglo V a.C., véase Fowler 2006, p. 36. 
A diferencia de lo dicho acerca de las genealogías, la presencia de catálogos es un elemento que acerca la Biblioteca a gran parte de la producción de época tardohelenística e imperial; basta con pensar en las listas que encontramos en la segunda parte de las Fábulas de Higino ${ }^{3}$ o en los fragmentos de papiros estudiados por Monique van RossumSteenbeek ${ }^{4}$.

Normalmente se hace una primera distinción entre listas que han sido compiladas a partir de una obra literaria precedente (por ejemplo, el catálogo de los argonautas) y otras que recogen informaciones de proveniencia varia $^{5}$. A su vez, en el primer caso, raramente una lista se limita a reproducir un texto más antiguo, sino que por lo general lo amplía, añadiendo otros héroes o recordando el nombre de los padres, de las madres o de las ciudades de los personajes que ya aparecían nombrados en su modelo literario. Solo con fijarnos en algunas listas tardías de los jefes aqueos que fueron a Troya, como la de Higino, es ya posible darse cuenta de cuántas informaciones se podían añadir al texto homérico $(F a b .97)^{6}$. A veces el autor de la lista podía simplemente sacar estas informaciones de otras fuentes literarias, pero hay muchos casos en los que la rareza de las informaciones nos hace dudar de que se puedan considerar originadas en verdaderas tradiciones míticas y, a su vez, nos hace sospechar que quizá se trate, en cambio, de creaciones eruditas.

No obstante, tenemos que considerar que los antiguos podían leer muchas más cosas que nosotros y que, por tanto, nunca se puede excluir con absoluta seguridad la posibilidad de que el autor de una lista haya encontrado nombres e informaciones en una obra literaria que hoy está perdida. Sin embargo, hay algunos catálogos que suscitan la legítima sospecha de que fueron enriquecidos con nombres ficticios, y uno de los más sospechosos es, seguramen-

\footnotetext{
${ }^{3}$ Aunque se pueden encontrar listas también en la parte narrativa de las Fábulas, los catálogos se concentran entre las fábulas 221-277.

${ }^{4}$ van Rossum-Steenbeek 1998, pp. 119-154.

${ }^{5}$ van Rossum-Steenbeek 1998, pp. 119-120.

${ }^{6}$ Catálogos de los aqueos que lucharon en Troya se encuentran también en otras obras: Apollod., Ep. III 11-14; Dict. I 17. Estas listas se inspiran en Hom., Il. II 484-759, aunque cada una presenta sus peculiaridades. La lista de Higino, por ejemplo, nombra también las madres de los jefes aqueos, ausentes del catálogo homérico y de las cuales, además, muchas nunca son mencionadas en el poema.
} 
te, la lista de los pretendientes de Penélope transmitida por un manuscrito de Jerusalén, que conserva fragmentos de la Biblioteca, los llamados Fragmentos sabaíticos ${ }^{7}$.

Aunque el texto presenta abundantes corrupciones, el manuscrito con los extractos de Apolodoro conserva un catálogo que consta del extraordinario numero de 129 pretendientes, frente a los 14 mencionados por Homero. Se observan algunas repeticiones e incongruencias: en particular, el número de los pretendientes que el manuscrito ofrece antes de cada uno de los grupos en los que el catálogo está dividido ${ }^{9}$ no corresponde a la suma total de los nombres que, de hecho, están citados ${ }^{10}$. Además, como veremos, en el manuscrito se encuentran muchos nombres sospechosos, dado que no están atestiguados como apelativos de persona en ningún otro texto antiguo.

A pesar de las posibles corrupciones y repeticiones, la diferencia con respecto a los catorce nombres citados en la Odisea es lo bastante grande como para suscitar preguntas sobre las fuentes de donde el compilador podría haberlos sacado. Estas preguntas se vuelven aún más justificadas si tenemos en cuenta que los pretendientes de Penélope, a diferencia de los de Helena $^{11}$, son personajes que no tienen una verdadera personalidad mítica fuera de la Odisea y, en consecuencia, es difícil imaginarse que el autor de la lista pudiera encontrar todos estos nombres en obras independientes de

\footnotetext{
${ }^{7}$ Algunos extractos de la Biblioteca sobre la saga troyana, en gran parte perdida en nuestra tradición manuscrita directa, se encuentran entre los folios 114a-125b del códice SabbaiticusHierosolymitanus 366 (Jerusalén, Patriarchike Bibliotheke, Hagiou Saba 366). La noticia del descubrimiento de estos fragmentos, seguida por su primera edición, fue publicada por Papadopulos-Kerameus en 1891. En ese mismo año Wagner 1891, el editor de la Biblioteca, publicó un estudio sobre este texto. Sobre este manuscrito véase Papathomopulos 1973.

${ }^{8}$ Véase infra.

${ }^{9}$ Los pretendientes aparecen distribuidos según el lugar de su procedencia: Duliquio, Zacinto, Ítaca y Samos, como en Hom., Od. XVI 245-254.

${ }^{10}$ El manuscrito afirma que desde Duliquio llegaron $57\left(v \zeta^{\prime}\right)$ pretendientes, pero nombra 53; desde Samos numera y nombra $23\left(\kappa \gamma^{\prime}\right)$, desde Zacinto dice que llegaron $44\left(\mu \delta^{\prime}\right)$, pero nombra solamente 41; desde Ítaca numera y nombra 12 (1 $\left.\beta^{\prime}\right)$; cf. Carrière 1991, pp. 292-293.

${ }^{11}$ La lista de los pretendientes de Helena se encuentra ya en el Catálogo de las mujeres de Hesíodo (fr. 196-204 M.W.). A diferencia del catálogo de los pretendientes de Penélope, en este caso los personajes nombrados son los héroes protagonistas de la saga troyana. Por tanto, esta lista podía servir como una especie de preludio a la guerra; véase Cingano 2005.
} 
Homero. Tampoco tenemos ninguna otra obra mitográfica, ningún escolio ni ningún fragmento de papiro que conserve otro elenco de los nombres de los pretendientes. El único testimonio que podemos comparar con el fragmento de Apolodoro es el papiro de Oxirrinco P.Oxy. LIII 3702, que contiene varias listas mitológicas (de los aqueos que fueron a Troya, de los argonautas, etc.), entre las cuales hay una de los pretendientes de Penélo$\mathrm{pe}^{12}$. Este papiro se limita dar los números de cuántos pretendientes provenían respectivamente de Ítaca, Samos, Duliquio y, probablemente, Zacinto, sin ofrecer una lista de sus nombres. El número de los pretendientes, al parecer, era un tema tratado también por Aristarco de Samotracia, que contaba ciento ocho pretendientes (Sch. Hom., Od. XVI 245). Tanto el cálculo de Aristarco como el texto reconstruido por los editores del papiro parecen seguir fielmente el cálculo que se puede extraer de los versos de Homero $\mathrm{y}$, en particular, del pasaje del libro 16 en el que Telémaco informa a Ulises de cuántos pretendientes, repartidos según sus proveniencias, están en su casa (Od. XVI 245-254).

En principio, entonces, un caso como este, en el que no existía una verdadera fuente literaria de donde el compilador pudiera obtener los nombres, podría llevar a pensar que esta clase de listas estuvieran construidas de manera totalmente arbitraria ${ }^{13}$. Sin embargo, en este artículo mi propósito es demostrar que también en casos de este tipo el compilador del catálogo tenía a su disposición y se valía de estrategias compositivas que le permitían fundamentar en la tradición los elementos que creaba ${ }^{14}$.

\section{Primera estrategia: la interpretación de Homero}

En primer lugar, podemos apreciar que el elenco de los catorces nombres mencionados por el poeta podía ser enriquecido gracias a la interpretación del mismo texto homérico, es decir, sin salir de la «tradición». De hecho, el escolio que nos transmite la referencia de Aristarco nos invita a creer en la existencia de otros cálculos del número de los pretendientes, pues de otro modo

12 Véase infra.

13 Esta era la opinión de Wagner 1891, p. 419.

${ }^{14}$ Un caso muy diferente es el de Dictis, que se proponía reescribir las tradiciones troyanas de una manera diferente del relato de Homero. Según este autor los pretendientes eran solo treinta (VI 6). 
no se explicaría el énfasis que pone el escoliasta en subrayar el acuerdo entre el filólogo alejandrino y los versos de Homero ${ }^{15}$. Probablemente Aristarco no habría perdido el tiempo en ofrecer un cálculo que todo el mundo hubiera podido hacer a partir del texto de Homero, a no ser que hubieran existido otros cálculos que, según el filólogo alejandrino, no encontraban legitimidad en el poema homérico. Y una confirmación de esta sospecha se puede encontrar, precisamente, en el mismo papiro del que hemos hablado antes: si el testimonio que ofrece el papiro parece seguir fielmente a Homero, ello se debe en buena medida a que la reconstrucción de su texto y las conjeturas de los editores se han basado, precisamente, en el pasaje de la Odisea del que hemos hablado antes. Sin embargo, esta reconstrucción contrasta con el análisis detenido de los restos de escritura que el papiro conserva. El número de los pretendientes que llegan desde Zacinto, que en el papiro se lee solo parcialmente (col. ii, 1. 34), y que el primer editor había reconstruido como una $\kappa$, es decir, «veinte», en realidad no puede corresponder a esta cifra, dado que los restos de tinta visibles no parecen compatibles con la $\kappa$. Por tanto, la cifra que el papiro ofrecía no parece corresponder al número que se encuentra en el poema homérico (véase Fig. 1 y Fig. 2$)^{16}$.

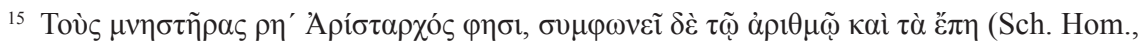
Od. XVI 245).

${ }^{16}$ La editio princeps (Haslam 1986) ofrece la lectura $\dot{\varepsilon}[\kappa$ Z $\alpha \kappa v ́(v) \mid \theta] \mathrm{ov} \bar{\kappa}$, que pone en duda van Rossum-Steenbeek (1998, pp. 312-313), quien descarta sin lugar a dudas que la cifra pueda, de hecho, leerse como $\kappa$. El número, pues, que por eliminación solo puede ser el de los pretendientes de Zacinto, no puede ser el mismo que el de Homero. La autora propone como posibles alternativas una beta, una theta o una omicron. Sin embargo, a la vista de la fotografía del papiro publicada en la base de datos P.Oxy. Online (consultada el 27 de mayo de 2020), ninguna de estas lecturas parece consistente con los restos de escritura conservados. En nuestra opinión los trazos, uno descendente y otro ligeramente redondeado ascendente, parecen del todo compatibles con una alfa. Quedando pendiente la confirmación mediante la autopsia del papiro, nos parece distinguir, a la izquierda de la parte superior de la letra en cuestión, restos de tinta que no pueden corresponder a la upsilon precedente, sino quizá a una letra entre ambas, correspondiente a la cifra de las decenas (el espacio disponible permitiría quizá leer $\overline{\imath \alpha}$, es decir, 'once', o quizá más probablemente $\overline{\kappa \alpha}$, 'veintiuno').
} 


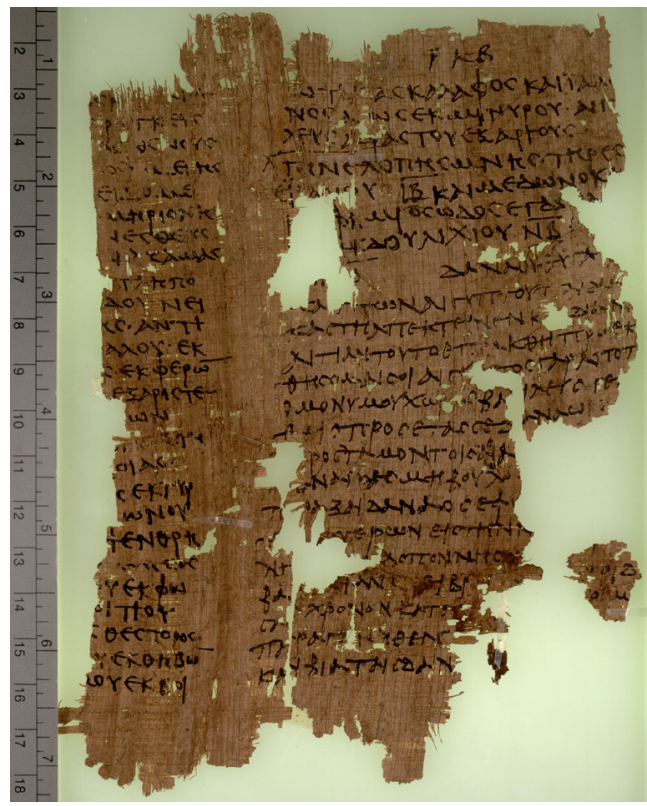

Fig. 1: P.Oxy. LIII 3702. Cortesía de la Egypt Exploration Society y del University of Oxford Imaging Papyri Project. (C) The Egypt Exploration Society. Contenido protegido por la ley de copyright del Reino Unido / Contents protected by UK copyright law.

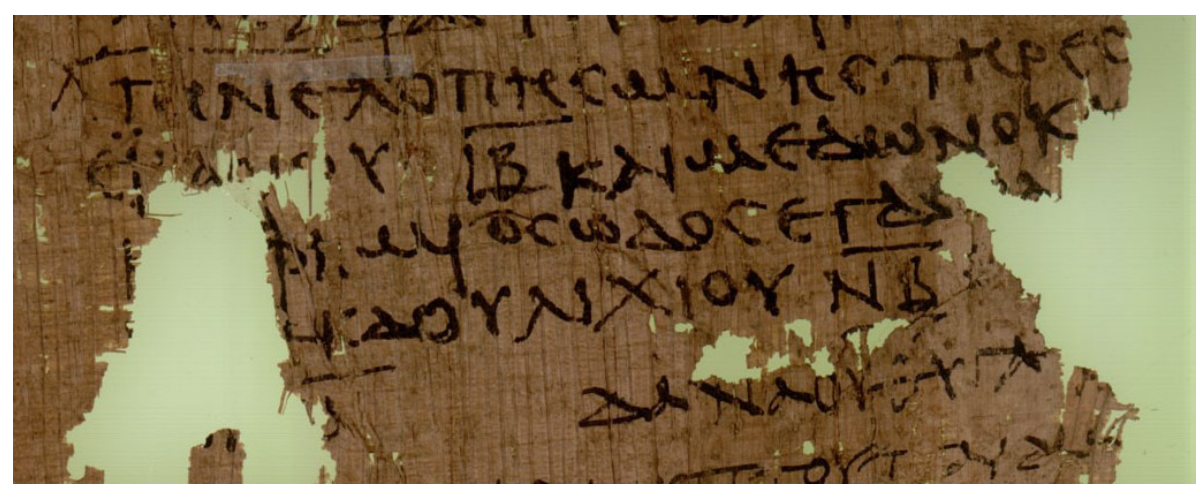

Fig. 2: Detalle del P.Oxy. LIII 3702 donde se aprecia el catálogo de los pretendientes de Penélope. Cortesía de la Egypt Exploration Society y del University of Oxford Imaging Papyri Project. (C) The Egypt Exploration Society. Contenido protegido por la ley de copyright del Reino Unido / Contents protected by UK copyright law. 
La verdad es que el mismo texto de Homero podía dar pie a interpretaciones diferentes. Cuando Telémaco enumera los pretendientes a su padre, a veces añade el número de los sirvientes que los acompañan, que, obviamente, no están incluidos en el número de los galanes ${ }^{17}$. Sin embargo, en el caso de los pretendientes de Ítaca, Telémaco menciona también al heraldo Medonte y al «divino aedo», es decir, Femio (Od. XVI 252). Estos dos personajes no son simples sirvientes como los otros. De hecho, en el momento de la venganza la ira de Ulises habría recaído también sobre ellos, como sobre todos los demás pretendientes, si no fuera por la intervención de Telémaco (XXII 330-380). Esto les confiere un estatuto ambiguo que, pese a lo extraño que nos pueda parecer, podía legitimar la idea de que Medonte y Femio también formaban parte de los pretendientes y, por lo tanto, podía crear dudas sobre el número de este grupo.

De hecho, esto es lo que pasa en el papiro ya mencionado: la primera entrada de la lista consta de doce $(1 \beta)$ pretendientes (seguramente de Ítaca,

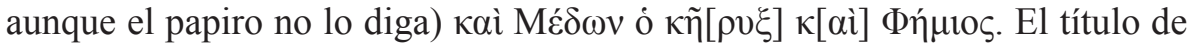

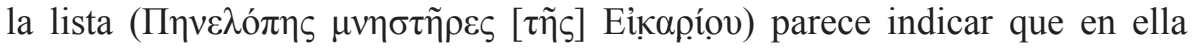
estén incluidos solo los pretendientes, y no todo su séquito, como en la Odi$\operatorname{sea}^{18}$, y que por lo tanto también Medonte y Femio deban ser considerados como integrantes de este grupo.

La comparación con otras fuentes nos muestra que la lista del papiro no es un caso completamente aislado, sino que se basa en una interpretación del texto de la Odisea que podía ser compartida por otros autores. Medonte es nombrado entre los pretendientes por Penélope en la primera de la Heroidas de Ovidio (I 91), un autor que, como ha demostrado Cameron, conocía y utilizaba instrumentos como listas y catálogos ${ }^{19}$, y lo mismo sucede con $\mathrm{Fe}$ mio: aunque existía una tradición que lo aproximaba al aedo que Agamenón dejó encargado de vigilar a su mujer durante su ausencia y le atribuía la misma función con respecto a Penélope (Sch. Hom., Od. III 325) ${ }^{20}$, podía tam-

17 Telémaco nombra seis sirvientes que acompañan a los pretendientes de Duliquio (Hom., Od. XVI 247) y dos sirvientes que cortan la carne con los pretendientes de Ítaca (XVI 253).

${ }_{18}$ De hecho, en el papiro no se hace mención de los otros ocho servidores nombrados por Telémaco.

${ }_{19}$ Cameron 2004, pp. 261-268.

${ }^{20}$ Otro escolio a la Odisea (I 327) atribuye a Femio un papel favorable a Ulises: Femio habría cantado los Nostoi de los aqueos para hacer que Penélope recordara a su esposo. 
bién ser considerado un pretendiente, como muestra un escolio a Elio Arístides (XXIV 5) ${ }^{21}$.

También en el catálogo de Apolodoro podemos reconocer la presencia de estos personajes: el nombre de Medonte aparece en el texto del manuscrito de Jerusalén (1. 1150) ${ }^{22}$. El hecho de que sea insertado entre los pretendientes de Duliquio y no entre los de Ítaca, como hace pensar el texto de la Odisea, no debe sorprendernos, ya que la manera en la que los nombres se distribuyen sigue criterios que a nosotros nos parecen desconcertantes: por ejemplo, encontramos un Ítaco entre los pretendientes de Samos (1. 1155) y un Duliquieo entre los de Ítaca (1. 1166).

El nombre de Femio no está atestiguado en el manuscrito, pero es la corrección propuesta por Kerameus, el primer editor de los Fragmentos Sabaíticos, para corregir la forma $\Phi \rho \varepsilon ́ v ı \varsigma$, que no está atestiguada en ningún otro texto ni como nombre de persona ni como sustantivo, y que se repite dos veces en la lista de los pretendientes de Zacinto (1. 1158; 1. 1161).

La presencia segura de Medonte y, si aceptamos la propuesta de Kerameus, la de Femio muestran cómo un primer procedimiento para enriquecer el catálogo podía consistir en una interpretación del texto homérico que a nosotros puede parecernos tendenciosa, pero que, sin embargo, era compartida por autores de la relevancia de Ovidio y que, por tanto, se puede considerar como una verdadera variante mítica. El límite, pues, entre tradición, interpretación y creación libre aparece aquí como muy sutil.

El caso de Medonte y Femio nos invita a buscar las trazas de otras posibles estrategias utilizadas para enriquecer la lista de los pretendientes que tengan un punto de apoyo en el texto homérico. En este sentido me parece un detalle digno de interés el hecho de que en el catálogo se encuentren cuatro nombres que se pueden relacionar con los padres de los pretendientes nombrados en el

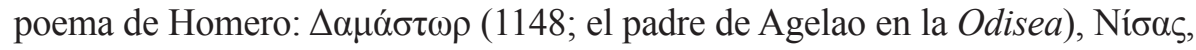
que Carrière, seguido por Papathomopulos, ha corregido en Níøos (1159; nom-

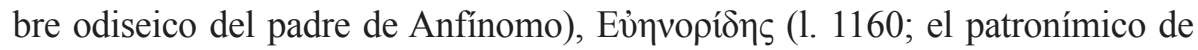

21 Otras tradiciones sobre este personaje nos confirman que Femio podía parecer una figura lo bastante noble como para aspirar a Penélope: Higino (81) lo considera un pretendiente de Helena.

22 La referencia a la línea del texto se basa en Papathomopulos 2010. 
Leócrito en el poema ${ }^{23}$ ) y Пó $\lambda \cup \beta o \varsigma$, que aparece dos veces en la lista (1. 1160; 1. 1164). En la Odisea Пó $\lambda v \beta$ os es el nombre de un pretendiente mencionado dos veces (XXII 243, 284), lo que explica su presencia en la lista de la Biblioteca, pero es también el padre de Eurímaco, y con este valor es nombrado hasta ocho veces (I 399; II 177; XV 519; XVI 345, 434; XVIII 349; XX 359; XXI 320). La diferencia entre los dos personajes no es muy clara y el hecho de que lleven el mismo epíteto, $\delta \alpha i ̂ ́ \varphi \rho \omega v^{24}$, puede sugerir una identificación entre los dos, como efectivamente se encuentra en muchos índices de nombres que siguen a las ediciones y las traducciones modernas de la Odisea ${ }^{25}$. No tenemos testimonios antiguos que nos permitan comprobar si los dos eran considerados como personajes diferentes o si se veían como la misma persona, pero podemos observar que esta segunda interpretación pudo ofrecer al compilador un recurso más para enriquecer su catálogo. En efecto, el hecho de que el padre de Eurímaco fuese a su vez uno de los galanes podía legitimar las sospechas de que también otros de los padres de los pretendientes lo fuesen. De esta forma

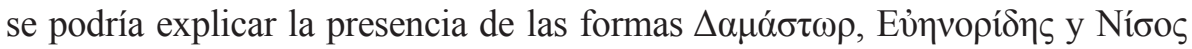
en el catálogo de Apolodoro.

Sin embargo, el hecho de que no todos los padres mencionados en el poema se encuentren también en el catálogo de Apolodoro parece contrastar con la hipótesis aquí avanzada. En realidad, la lista, por lo menos en la forma en la que nos ha llegado, muestra la misma falta de sistematicidad también en relación a los nombres de los propios pretendientes mencionados en la Odisea: de los 14 pretendientes faltan tres, entre ellos Eurímaco, quizá el más importante $\mathrm{y}$, aunque sea probable que los tres nombres hayan desaparecido por corrupciones del texto, las soluciones propuestas por los editores para restituirlos son problemáticas y no siempre aceptadas por la comunidad científica $^{26}$. De la misma manera pueden haber desaparecido otros nombres, pero

${ }^{23}$ En la Odisea, así como en el catálogo, no se encuentra el nombre de Eỏń

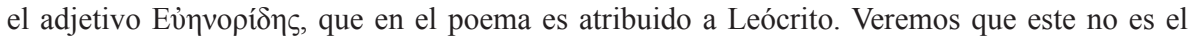
único caso en el que el compilador utiliza en su lista adjetivos homéricos como si fueran nombres propios. En este caso no hay dudas sobre el hecho de que con este nombre el compilador querría referirse al padre de Leócrito, porque este adjetivo se encuentra otra vez solo en Quinto de Esmirna (IV 334).

${ }^{24} \Delta \alpha$ aî́p $\omega v$ aplicado al padre de Eurímaco: XV 519; aplicado al pretendiente: XXII 243.

${ }^{25}$ Esta identificación ya se encuentra en el diccionario de Smith 1849, p. 449.

${ }^{26}$ Papathomopulos (2010) acepta las conjeturas propuestas por Carrière (1991, pp. 239-294), pero Scarpi (1996, pp.408-409), que conoce y cita a propósito de este catálogo a Carrière 
tenemos que considerar que, si el texto homérico nos permite pensar que en el grupo de los galanes se encontraban también algunos padres de los pretendientes, al mismo tiempo nos obliga a excluir la posibilidad de que todos los padres estuvieran en la corte de Ulises. En la Odisea, Eupites, el progenitor de Antínoo, el más nombrado de los padres en el poema homérico y ausente del catálogo de la Biblioteca, no podía ser uno de los galanes, dado que sigue vivo después de la matanza cumplida por Ulises y guía el sucesivo intento de venganza de los varones de Ítaca que habían perdido algún pariente en la matanza (XXIV 422 ss.). Al final, muere antes de la intervención pacificadora de Atenea (XXIV 523).

La posibilidad de que la presencia en la lista de cuatro nombres de padres de pretendientes no sea una coincidencia, sino que dependa de una interpretación del texto homérico, no puede, por tanto, ser descartada.

\section{Segunda estrategia: el centón homérico}

Si bien la interpretación del texto homérico podía ofrecer un apoyo para enriquecer la lista, es seguro que este no era el único procedimiento utilizado. De hecho, por ahora hemos conseguido rastrear un posible origen solo para cinco o seis de los nombres de la Biblioteca, con lo que nos quedamos muy lejos de los 129 citados por el compilador. Es probable que muchos de estos hayan sido «inventados», cosa que no tiene que asombrarnos, dado que la posibilidad de la invención de nombres y de personajes era algo admitido y ya atribuido al mismo Homero por parte de Aristarco ${ }^{27}$. Sin embargo, cuando alguien inventa, rara vez lo hace creando de la nada. Y también en el inventar nombres podían existir métodos compartidos por las personas que los inventaban y tradiciones precedentes en las que podían inspirarse. La posibilidad de que el compilador recogiera tradiciones que nosotros no conocemos nunca podrá ser descartada con seguridad, como muestra el caso de las inscripciones de los frescos que forman el llamado «Ciclo de la Odisea de via Graziosa $»^{28}$. La pintura, que muestra el encuentro entre tres compañeros

(p. 667), publica el texto de Wagner sin sus correcciones.

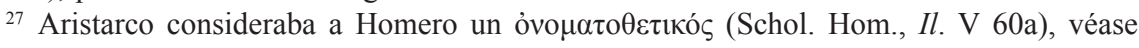
Nünlist 2019, pp. 69-70.

${ }^{28}$ Denominados también «Odyssey Landscapes», son frescos con escenas de la Odisea conservados en los Museos Vaticanos, descubiertos a mediados del siglo XIX en la ladera 
enviados por Ulises y los lestrigones, lleva inscripciones con los nombres de los tres (Euríbates, Antíloco y Anquíalo), aunque en la Odisea estos personajes sean anónimos (X 100-102). Si el nombre de Euríbates, es decir, 'el de largas zancadas', es atribuido a un heraldo de Ulises en los dos poemas homéricos (Il. II 184; Od. XIX 247), los otros dos, Antíloco y Anquíalo, no aparecen mencionados por Homero en el séquito del héroe. Por eso se había pensado que podían ser el producto de una simple invención ${ }^{29}$; sin embargo, se ha notado que uno de los dos nombres, Antíloco, se encuentra también en las Quilíades de Tzetzes, entre los compañeros que se libran de ser comidos por el Cíclope (X 885), y que en la línea precedente el erudito bizantino nombra un «Anfíalo» que podría ser identificado con el Anquíalo de la pintura. Por eso se ha supuesto la existencia de una verdadera tradición común, probablemente debida a algún autor helenístico o quizás a una fuente aún más antigua. De hecho, según la descripción de Pausanias, un compañero de Ulises se llamaba Anquíalo en la Iliupersis de Polignoto (X 27.3) ${ }^{30}$.

Este caso nos invita, pues, a tener en cuenta la posibilidad de que también algunos de los nombres que encontramos en la lista de Apolodoro hubieran podido ser atribuidos a los pretendientes ya en época bastante antigua: en efecto, la idea de dar un nombre a los personajes anónimos de la Odisea es tan antigua como la propia mitografía, dado que ya Ferécides daba nombre a los compañeros comidos por Escila (EGM 144), tal como examinaremos en el próximo párrafo.

Sin embargo, aún hay otra posible explicación para los nombres de los compañeros de Ulises en el ciclo pictórico de via Graziosa, que puede también valer para muchas otras listas de personajes que tienen que ver con la saga troyana. Esta explicación, como veremos, ofrece un claro ejemplo de cómo la invención se puede también basar en el conocimiento de la tradición. Acabamos de señalar cómo los dos compañeros de Ulises de la pintura llevan los nombres de otros personajes nombrados en la Ilíada y en la Odisea, lo que ha llevado a pensar que el pintor tomase estos nombres de otros pasajes del poema y los atribuyera a los dos compañeros de Ulises ${ }^{31}$.

occidental del Cispio, en Roma, y que datan probablemente de en torno al año 50 a. C.; véase Lowenstam 1995; Coarelli 1998; O'Sullivan 2007.

${ }^{29}$ Müller 1913, p. 146

${ }^{30}$ Sobre toda esta cuestión véase Lowenstam 1995, pp. 202-207.

31 Nogara 1907, p. 42. 
Esta consideración resulta aún más apropiada para el catálogo de los pretendientes, donde, si tomamos la edición de Wagner ${ }^{32}$, más fiel al texto del manuscrito que la de Papathomopulos, y tenemos en cuenta también las repeticiones, 69 de los 129 nombres se encuentran en los poemas homéricos, en otros pasajes. Al menos en un caso hay muchas pruebas que fundamentan la idea de que el compilador del catálogo eligió efectivamente algunos de los nombres a partir de la lectura de pasajes diversos de la propia Odisea.

En la lista encontramos los nombres de algunos personajes relacionados

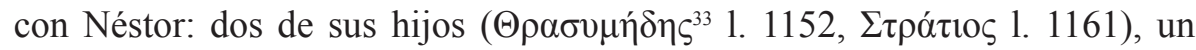

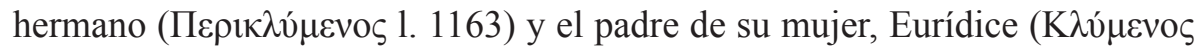

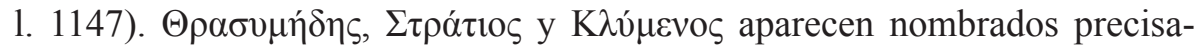
mente en el pasaje de la Odisea donde se describe el banquete organizado por Néstor para acoger a Telémaco (Od. III 439-452). Se podría pensar en una

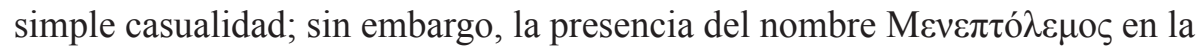
lista de Apolodoro (1.1147) revela que el compilador tenía realmente en mente este pasaje mientras elaboraba su catálogo. En efecto, como nombre de persona esta forma es muy rara ${ }^{34}$; sin embargo, es un epíteto que aparece muchas veces en Homero y en la poesía griega. En la Odisea se encuentra solo una vez, en el pasaje del banquete de Néstor del que hemos hablado antes (III 448), donde precisamente se refiere a $\Theta \rho \alpha \sigma v \mu \eta \dot{\delta} \delta \eta$ s. Además, este no es el único nombre presente en el catálogo que se puede poner en relación con los pasajes de la Odisea que mencionan miembros de la familia del rey

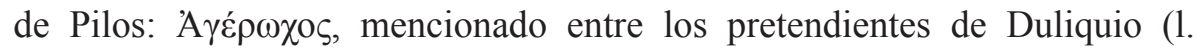
1150 ), es una forma que no he podido encontrar como nombre de persona en ninguno de los repertorios consultados, pero que como adjetivo aparece ocho veces en Homero, de las cuales una sola en la Odisea, donde se refiere pre-

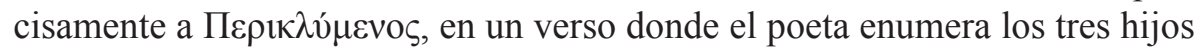
de Neleo y Cloris (XI 286).

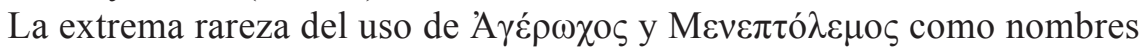
de persona nos asegura, con buena probabilidad, que el compilador, efectivamente, había leído los dos pasajes de la Odisea que nombran a los miem-

32 Wagner 1894, pp. 233-234.

${ }^{33}$ Esta forma es una corrección muy probable de $\Theta \rho \alpha \sigma u \mu i ́ \delta \eta \varsigma$, forma nunca atestiguada, propuesta por Papadopulos-Kerameus (1891) y aceptada por todos los editores posteriores.

${ }^{34}$ Está atestiguado una sola vez en todo el LGPN (Lexicon of Greek Personal Names) y en un pasaje de Pausanias (VI 14.13). 
bros de la familia de Néstor y que decidió utilizarlos para compilar su lista de los galanes.

Hay un argumento aún más fuerte en apoyo a esta hipótesis: el manuscrito presenta también la forma $\mathrm{N} \varepsilon \sigma \tau o \rho i ́ \delta \eta \varsigma$. Aunque algunos editores hayan propuesto algunas correcciones ${ }^{35}$, esta forma se encuadra perfectamente en nuestra lista y demuestra un interés por la familia del rey de Pilo.

De hecho, este no es el único caso que puede legitimar la sospecha de que algunos de los nombres del catálogo procedan de personajes homéricos que pertenecen a un mismo grupo: entre los nombres citados por Apolodoro hay tres formas que corresponden precisamente a tres de los hijos de Antenor

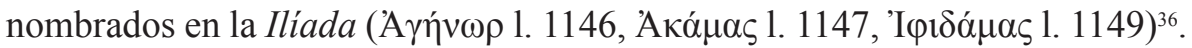
Además, hay dos formas muy raras que se pueden explicar como la corrupción de los nombres de otros dos hijos de Antenor en el poema de Homero

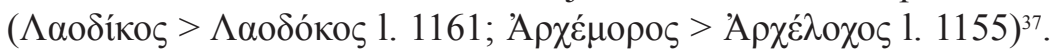

El caso de los nombres que se pueden relacionar con Néstor o con Antenor nos permite, por tanto, detectar una segunda estrategia que el compilador parece haber utilizado para elaborar la lista de los pretendientes. Esta segunda estrategia se puede relacionar con la práctica literaria de los centones. Si asumimos que el centón constituye un verdadero género literario del mundo antiguo, claramente no podremos incluir nuestro catálogo en este tipo de producción, por el simple hecho de que no se trata de una composición poética formada por porciones de los versos de Homero $^{38}$. Sin embargo, la manera en la que el autor utiliza los nombres de la familia de Néstor y de los hijos de Antenor para incluirlos en el catálogo como denominaciones de pretendientes se adapta perfectamente a la definición más general de centón, entendido como una écriture que se puede realizar de muchas formas, incluida la prosa, y que consiste en la selección y trasferencia de frases o sintagmas de un texto para componer otro sobre un tema diferente ${ }^{39}$.

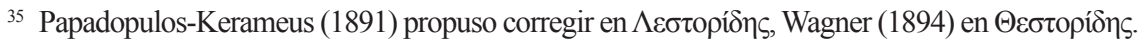

${ }^{36}$ También Пó $\nu$ ßos, que aparece en la lista de los galanes (1. 1160), es el nombre de un hijo de Antenor, pero, como hemos dicho, es más probable que aquí se refiera al pretendiente de Penélope o al padre de Eurímaco.

37 Sobre estas correcciones véase infra.

${ }^{38}$ Cf. Usher 1998, pp. 1-2.

39 Verweyen \& Witting 1991, 172 «Cento is not a generic term but an écriture — such as parody, travesty, contrafacture, and pastiche - which can be realized in a lyric and in an epic 
El hecho de que seleccionara nombres que pertenecen a un mismo grupo de personajes nos puede llevar a pensar que el compilador hubiera ya elaborado otras listas homéricas. No obstante, la presencia de epítetos parece excluir la posibilidad de que simplemente utilizase estas listas para rellenar su catálogo y más bien nos invita a suponer una lectura directa de los pasajes seleccionados del texto de homero también en la fase compositiva ${ }^{40}$.

\section{Los nombres no homéricos}

Aunque una gran parte del catálogo parezca construida como una recopilación de nombres y adjetivos extraídos directamente de los poemas homéricos, sin embargo, quedan aún otros 60 nombres que no se pueden simplemente explicar de esta manera. Entre ellos encontramos formas tan raras que para nosotros son verdaderos hapax: el manuscrito transmite 28 formas que no están atestiguadas en otros textos ni como nombre de persona ni como sustan-

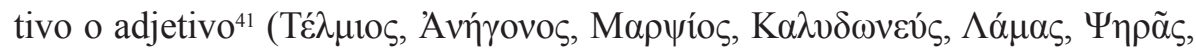

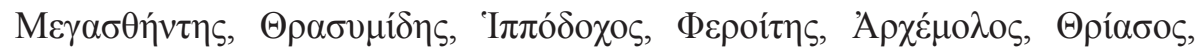

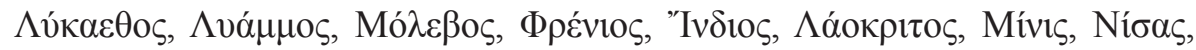

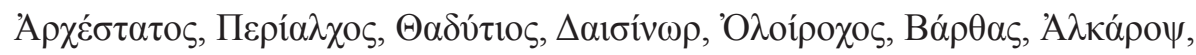

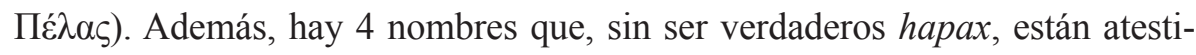

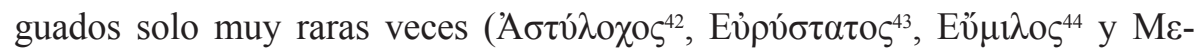

form as well as in the prose of political treatises and the literary essay, even in dramatic form. This écriture consists in the selection of sentences or syntagms from one or several texts and transferring them unalteredly into a new text with a different topic».

${ }^{40}$ Hay otros casos en los que podemos pensar que el compilador extrajo algunos nombres directamente de un mismo pasaje del poema. Por ejemplo, en la lista encontramos los nombres de dos compañeros de Ulises nombrados en la Odisea, Eủ el segundo, las dos veces que aparece mencionado en el poema, lo hace siempre en pareja con

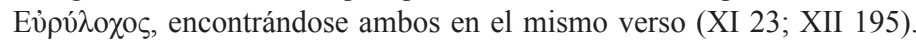

${ }^{41}$ Hemos considerado aquí solo las formas transmitidas por el manuscrito de Jerusalén, sin las correcciones de los editores.

${ }^{42}$ Se atestigua solo dos veces en $L G P N$, y nunca en el $T L G$.

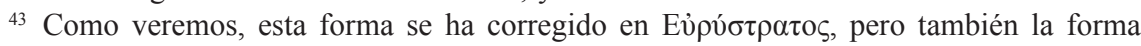

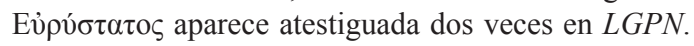

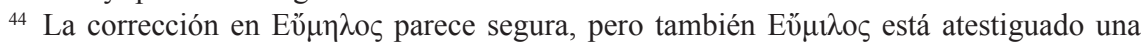
vez en $L G P N$. 
$v \varepsilon \pi \tau$ ó $\lambda \varepsilon \mu \varsigma^{45}$ ), 7 que están atestiguados únicamente como adjetivos ("A $\gamma 10 \varsigma$,

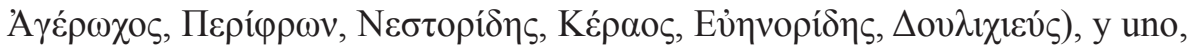
Прó $\mu$ os, que se encuentra solamente a partir de la época bizantina ${ }^{46}$.

Un número tan grande de hapax se debe seguramente en parte a corrupcio-

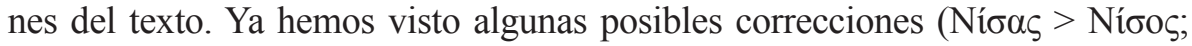

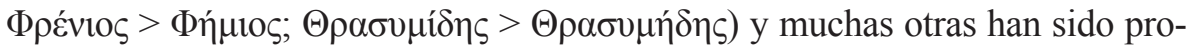
puestas y, a veces, integradas en el texto por los editores. Algunas parecen fonéticamente muy probables y restituyen otras formas homéricas $\left(E \ddot{\mu} \mu \imath \lambda_{0} \varsigma>\right.$

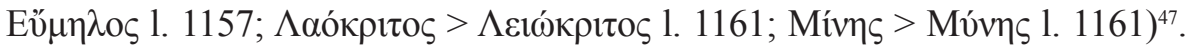

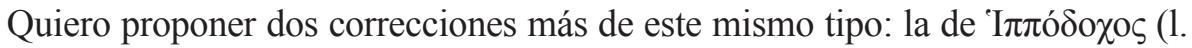
1154), nombre nunca atestiguado, en 'I $\pi \pi \delta ́ \lambda o \chi o \varsigma$, que aparece en la Ilíada una vez como padre de Glauco (XVII 40) y otras siete como guerrero troyano hijo de Antímaco (VI 119, 197, 206; VII 13; XIII 309, 387; XVII 140), y la de $\Lambda \alpha o \delta$ í́ç (1. 1161), atestiguado como nombre mitológico una única vez, como

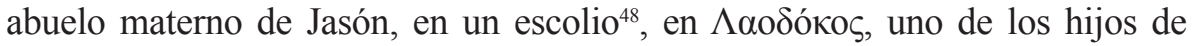
Antenor en la Ilíada, de los que hemos hablado en el apartado precedente.

Otras correcciones propuestas, o restituyen nombres de personas nunca

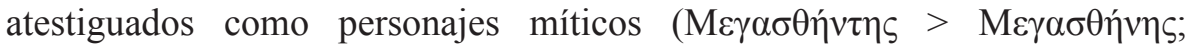
A $\rho \chi \bar{\varepsilon} \sigma \tau \alpha \tau o \varsigma>$ 'A $\rho \chi \dot{\varepsilon} \sigma \tau \rho \alpha \tau o \varsigma)$, o no son aceptadas por todos los editores. Entre estas últimas, algunas restituyen nombres que encajan perfectamente en un catálogo de los pretendientes y se pueden explicar a partir de las estrate-

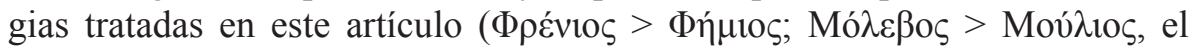

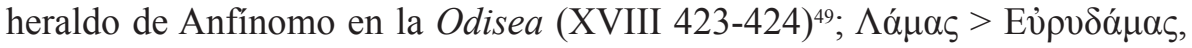

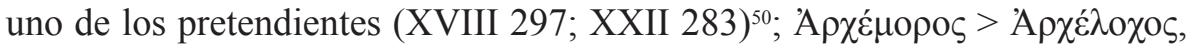
un hijo de Antenor, como ya hemos visto $)^{51}$.

${ }^{45}$ Cf. n. 34.

${ }^{46}$ Encontramos la forma Прó $\mu$ o como nombre de persona por primera vez en la época bizantina, donde es una variante de Прóßos; sin embargo, como adjetivo es una forma atestiguada ya en Homero. Por tanto, su presencia se puede explicar del mismo modo que la de los otros adjetivos incluidos en nuestra lista.

${ }^{47} \mathrm{He}$ incluido ya estas formas en el cálculo de los 69 nombres homéricos presentes en el texto de Wagner.

48 Sch. A.R. I $45-47 \mathrm{a}$

49 Propuesta por Papadopulos-Kerameus 1891.

${ }^{50}$ Propuesta por Carrière 1991.

${ }^{51}$ Hay otras correcciones fonéticamente muy plausibles que, sin embargo, no ayudan mucho a mejorar el texto: las formas reconstruidas por los editores $\Psi \nu \rho \tilde{\varsigma} \varsigma, \Delta \alpha \iota \sigma \eta ́ v \omega \rho, \Lambda v ́ \kappa \alpha 1 \theta 0 \varsigma$, 
Entre las formas de nuestra lista que no corresponden a nombres homéricos, hay también algunas que no están atestiguadas como nombre mitológico en ninguna otra fuente y que, por lo tanto, pueden parecer muy sospechosas. Sin embargo, no todas estas formas tienen que ser consideradas como el resultado de una corrupción del texto: tres adjetivos que hemos analizado en

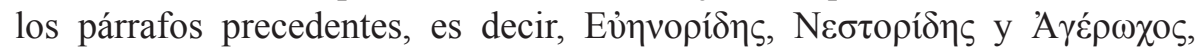
muestran cómo también formas nunca atestiguadas como nombres propios podían ser elegidas por el compilador, cumpliendo una verdadera estrategia compositiva. A estos adjetivos podemos añadir la forma Пврí $\varphi \rho \omega v$, nunca atestiguada como antropónimo, pero que en la Odisea es el epíteto utilizado comúnmente para referirse a Penélope. Ya Roscher creía que el nombre del pretendiente se había creado a partir del epíteto ${ }^{52}$.

Así pues, tampoco la naturaleza no mitológica de un nombre es un criterio seguro para distinguir lo que pertenece a la tradición de lo que es simple ficción. De hecho, tenemos que considerar que no siempre lo que a nosotros nos parece completamente ajeno al mundo de Homero debía parecérselo

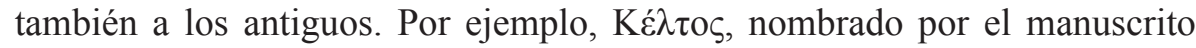
entre los pretendientes de Zacinto (1. 1163), a nosotros puede parecernos una

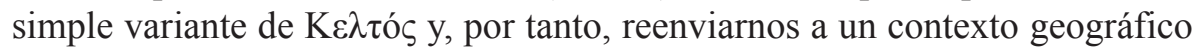
ajeno al mundo de los poemas homéricos; sin embargo, Quinto de Esmirna

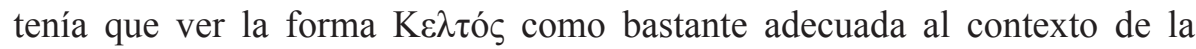
guerra Troyana, puesto que la insertó en su poema como nombre de un troyano matado por Neoptólemo (VII 611).

\section{El catálogo de los pretendientes y otras listas odisiacas}

La presencia de nombres raros no es una característica exclusiva de la lista de los pretendientes transmitida por los fragmentos de la Biblioteca y, por tanto, no se puede atribuir solamente a una falta de recursos del compilador frente al número enorme de nombres que tenía que proporcionar al lector. Formas igualmente raras se encuentran en las listas de compañeros de Ulises transmitidas por fuentes muy diferentes entre sí. Un nombre como MANTIXO

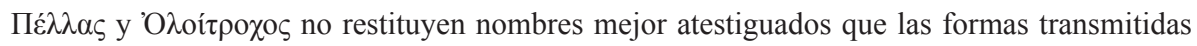

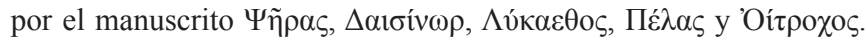

${ }_{52}$ Roscher 1902-1909, col. 1978. 
nunca atestiguado en los textos literarios y tampoco en el $L G P N$, aparece en tres «Homeric Bowls» que representan la escena de la transformación de los compañeros de Ulises en cerdos por obra de $\mathrm{Circe}^{53}$. En un texto muy diferente, como la lista de los seis compañeros comidos por Escila atribuida por un escolio (Sch. Hom., Od. XII 257) a Ferécides (EGM fr. 144), encontramos formas como 'A $\gamma \chi \mu o \varsigma$ o $\Sigma i ́ v \omega \pi o \zeta$, que tampoco están atestiguadas, o al menos no como nombres de persona ${ }^{54}$. Esta lista, con muy pocas diferencias, se encuentra también en Eustacio de Tesalónica.

Uno de los nombres mencionados por el escolio y por Eustacio, 'O $\rho \mu \varepsilon ́ v ı$,

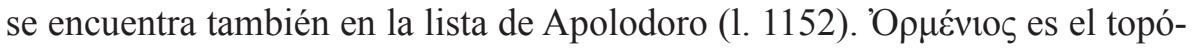
nimo de una ciudad de Tesalia mencionada ya en la Ilíada (II 734), pero como nombre de persona casi no está atestiguado ${ }^{55}$. Este no es el único nombre raro que aparece en el catálogo de los pretendientes y en otras listas de tema odisíaco. La forma $\Theta \varepsilon o ́ \varphi \rho \omega v$ es un adjetivo que antes de la época cristiana no parece haber sido muy común y que como antropónimo aparece solo trece veces en el $L G P N$ y ninguna en el $T L G$, pero se nombra entre los pretendientes de Zacinto y se conoce también por una didascalia de un «Homeric Bowl» que lo señala como uno de los compañeros de Ulises ${ }^{56}$.

Hay, por tanto, similitudes entre la lista de los pretendientes y los demás elencos que encontramos tanto en la tradición mitográfica como en contextos diferentes, como la iconografía sobre cerámica. Por eso, aunque una parte de las formas raras transmitidas por el manuscrito probablemente se hayan originado por una simple corrupción del texto, es legítimo pensar que algunas

${ }^{53}$ LIMC Mantichos 1; 2; 3.

${ }^{54} \mathrm{La}$ forma 'A $\gamma \chi \mu \mathrm{\jmath}$ s se encuentra solo en un fragmento de Eurípides ( $\left.f r .867\right)$, glosado por

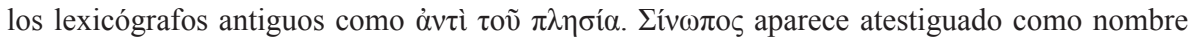
de un río de Sinope en los escolios y en el comentario de Eustacio a un verso de Dionisio el Periegeta (254).

${ }^{55}$ En el $L G P N$ no está atestiguado y en la literatura aparece solo dos veces: en el primer caso se encuentra inserto, una vez más, en una lista: la de los héroes abatidos por Deríades en Nono de Panópolis ( $D$. XXXII 186). El único verdadero personaje mítico que lleva este nombre es, muy probablemente, el rey epónimo de la ciudad de Tesalia. Diodoro llama Ormenio al padre de Astidamenia, muerto a manos de Heracles, rey de una ciudad anónima de la Pelósgide (IV 37.4). Podemos identificar esta ciudad con la Ormenio nombrada por Homero, si consideramos que en la Biblioteca el padre de Astidamenia (II 166), que aquí aparece llamado Amíntor, es el rey de Ormenio que mató Heracles (II 155).

${ }^{56}$ LIMC Theophron 1; 2. 
de ellas sean tradicionales, en el sentido de que pertenezcan a un acervo de nombres que parecían apropiados para un contexto mítico relacionado con la Odisea.

De hecho, existían con seguridad otras tradiciones literarias sobre el viaje de Ulises, como los Nostoi de Estesícoro, y es posible que Ferécides, un autor que se preocupaba especialmente por dar nombres a personas que antes no lo tenían ${ }^{57}$, por un lado utilizase estas fuentes, ${ }^{58} \mathrm{y}$ por otro ofreciera material a los siguientes compiladores de listas.

Así pues, es posible que también algunas de las formas no homéricas hayan sido introducidas en el catálogo según la lógica del centón y que, a su vez, las fuentes de esta composición no hayan sido solamente la Odisea y la Ilíada, sino además un corpus de textos épicos, mitográficos y eruditos que se referían también al contexto de la saga troyana ${ }^{59}$.

\section{Conclusiones}

Al final de este análisis podemos intentar responder a la pregunta de la que partíamos: de dónde pudo obtener el mitógrafo una lista tan larga de nombres de personajes que en la tradición literaria original eran anónimos. Ni la idea de una invención libre ni la posibilidad de una fuente literaria independiente de la Odisea pueden ofrecer, por sí solas, una explicación completa. La solu-

57 Forsdyke 1957, p. 143.

${ }^{58}$ Cameron (2004, p. 240) supone que la fuente de Ferécides para los nombres de los seis compañeros de Ulises comidos por Escila sean los Nostoi de Estesícoro.

${ }_{59}$ En este sentido es posible que la presencia de algunos nombres relativos a los otros hijos de Ulises no sea una simple coincidencia, sino que dependa de tradiciones diferentes de la homérica, originadas en la épica arcaica. Apolodoro nombra entre los pretendientes un 'A $\gamma p i o s$ (1. 1150), que en la Teogonía de Hesíodo Ulises engendra con Circe (Th. 1013), y un

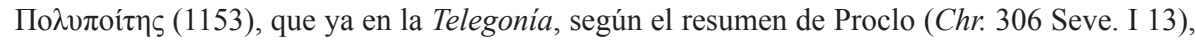
era hijo del héroe y de Calídice. Además, en el catálogo de los pretendientes encontramos

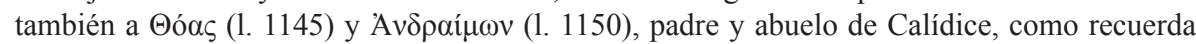
el mismo Apolodoro en el párrafo siguiente (Ep. VII 40). De todos modos, la referencia a los hijos no es segura y las dos formas se podían haber extraído sin más del texto homérico.

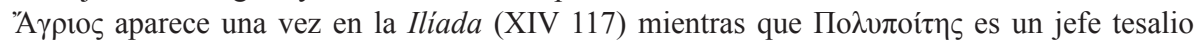
mencionado ochos veces por Homero (II 740; VI 29; XII 129, 182; XXIII 836, 844, 848). También $\Theta o ́ \alpha \varsigma$ y Av $\delta \rho \alpha i ́ \mu \omega v$ se pueden simplemente explicar como nombres homéricos, ya que se encuentran en el mismo verso del catálogo de las naves (II 638). 
ción se encuentra, probablemente, a medio camino entre estas dos opciones e implica el empleo de más de una única estrategia compositiva.

El propio texto de Homero ofrecía la posibilidad de interpretaciones originales sobre el posible papel desempeñado por los diversos héroes nombrados y, por tanto, podía permitir que se considerase como pretendientes también a personajes que, de una cierta forma, pertenecían al grupo de aquellos.

Este primer recurso, sin embargo, no podía ofrecer suficientes nombres como para completar un catálogo tan grande como el de los galanes, y de ahí que nuestro compilador parezca haber utilizado también otros nombres relacionados con el mundo de la Odisea, según una lógica que recuerda la de los centones. La fuente principal eran seguramente los poemas homéricos, pero hemos visto que existían nombres no homéricos que probablemente tenían una relación con el mito de Ulises, dado que se encuentran también en otros catálogos sobre esta temática.

Queda una última cuestión: si el compilador de este catálogo es el mismo autor que compuso la Biblioteca o si Apolodoro lo obtuvo de otra fuente. El único indicio para enfrentarse a esta cuestión es el que ofrecen las formas que hemos puesto en relación con la familia de Néstor. Una de estas formas era $\Sigma \tau \rho \alpha ́ \tau \imath o \zeta$, un hijo de Néstor en la Odisea que, sin embargo, no es mencionado en la lista de su descendencia en el primer libro de la Biblioteca (1.94). Si el mitógrafo hubiera conocido a fondo los pasajes homéricos relativos a Néstor, como implica nuestro catálogo, probablemente no se habría olvidado de

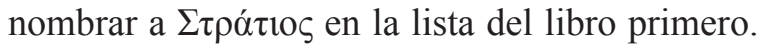

Aunque este argumento no resuelva del todo la cuestión, dado que se pueden encontrar también otras incoherencias parecidas entre diferentes partes de la Biblioteca ${ }^{60}$, las razones por las cuales el mitógrafo decidió introducir un catálogo tan largo en su obra resultan bastante claras. Obras eruditas que escenifican banquetes u otros contextos de diálogos de las elites culturales de la época imperial, como los Deipnosofistas, las Cuestiones convivales de Plutarco o las Noches áticas, nos muestran muchas veces cómo la capacidad de mencionar nombres míticos raros y desconocidos a los demás era algo

${ }^{60}$ La materia mítica que confluye en la Biblioteca es heterogénea y en una sección de la obra el mitógrafo podía seguir una tradición en contraste con otra utilizada en otra parte. 
muy importante y apreciado. El conocimiento de los nombres era una manera de afirmar la propia cultura en un contexto muy competitivo ${ }^{61}$.

En este sentido el catálogo de los pretendientes nos permite, también, reflexionar sobre el público de la Biblioteca. Muchas veces se ha subrayado la simplicidad de su prosa y la rapidez con la que se narran los relatos más importantes, y en consecuencia se ha visto en esta obra un simple repertorio para un público bastante amplio, que querría adquirir un conocimiento básico de los mitos tratados en las grandes obras del pasado griego. En realidad, la presencia de catálogos como este, así como la riqueza de variantes y de citas - a veces de autores raros - nos invita a pensar que el mitógrafo no se dirigía únicamente a este público más amplio. Su obra permite diferentes niveles de lectura que podían satisfacer tanto a personas de cultura relativamente básica como a los eruditos de los banquetes representados por Ateneo o Plutarco ${ }^{62}$.

\section{BIBLIOGRAFÍA}

Cameron, A. (2004): Greek Mythography in the Roman World, Oxford.

Carrière, J.-C. y Massonie, B. (1991): La Bibliothèque d'Apollodore. Traduite, annotée et commentée, Besanzón.

Cingano, E. (2005): «A Catalogue Within a Catalogue: Helen's Suitors in the Hesiodic Catalogue of Women (frr. 196-204)», en Hunter, R. (ed.), The Hesiodic Catalogue of Women. Constructions and Reconstructions, Cambridge, pp. 118-152.

Coarelli, F. (1998): «The Odyssey Frescoes of the Via Graziosa: A Proposed Context», PBSR 66, pp. 21-37.

Forsdyke, J. (1957): Greece Before Homer. Ancient Chronology and Mythology, Nueva York.

Fowler, R. L. (2006): «How to Tell a Myth», Kernos 19, pp. 35-46.

Fowler, R. L. (2013): Early Greek Mythography. Volume 2: Commentary, Oxford.

${ }^{61}$ Véase Johnson 2010, passim. El célebre interés de Tiberio por los nombres mitológicos (Suet., Tib. 70.3) es un ejemplo de cómo el poder nombrar también los personajes más raros seguramente fuera una cualidad muy apreciada entre los eruditos. Morgan (1998, pp. 82 ss.) ha demostrado que también en contextos escolares la capacidad de citar obras, autores y variantes raras podía ser un instrumento utilizado por los profesores para afirmar sus competencias.

${ }_{62}$ Fowler (2017) ha llegado a conclusiones parecidas, asumiendo que algunas de las informaciones más raras transmitidas por el mitógrafo se dirigían a un público de elite. Cameron (2004, pp. 253 ss.) ha mostrado cómo también los poetas podían utilizar obras mitográficas. 
Fowler, R. L. (2017): «Apollodorus and the Art of the Variant», en Pàmias J. (ed.), Apollodoriana. Ancient Myths, New Crossroads, Berlín-Boston, pp. 58-175.

Goldhill, S. (2009): «The Anecdote: Exploring the Boundaries Between Oral and Literate Performance in the Second Sophistic», en Johnson, W. A. \& Parker, H. N. (eds.), Ancient Literacies. The Culture of Readings in Greece and Rome, Oxford, pp. 96-113.

Haslam, M. W. (1986): «3702. Mythological Compendium», en Haslam, M. W. (ed.) The Oxyrhynchus Papyri vol. 53, Londres, pp. 31-40.

Johnson W. A. (2010): Readers and Reading Culture in the High Roman Empire. A Study of Elite Communities, Oxford.

Lowenstam, S. (1995): «The Sources of the Odyssey Landscapes», EMC 39, pp. 193-226.

Morgan, T. (1998): Literate Education in the Hellenistic and Roman Worlds, Cambridge-Nueva York-Melbourne.

Müller, F. (1913): Die antiken Odyssee-Illustrationen in ihrer kunsthistorischen Entwicklung, Berlín.

Nogara. B. (1907): Le nozze Aldobrandine, i paesaggi con scene dell'Odissea e le altre pitture murali antiche, Milán.

Nünlist, R. (2009): The Ancient Critic at Work: Terms and Concepts of Literary Criticism in Greek Scholia, Cambridge.

O'Sullivan, T. M. (2007): «Walking with Odysseus: The Portico Frame of Odyssey Landscapes», AJPh 128, pp. 497-532.

Papadopulos-Kerameus, A. (1891): «Apollodori bibliothecae fragmenta Sabbaticia», RhM 46, pp. 161-192.

Papathomopulos, M. (1973): «Pour une nouvelle édition de la Bibliothèque

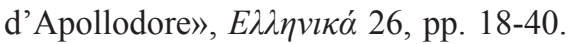

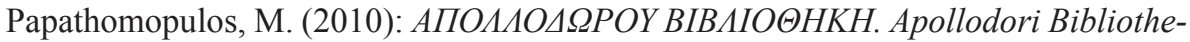
ca post Richardum Wagnerum recognita, Atenas.

Roscher, W. H. (1902-1909): Ausführliches Lexikon der griechischen und römischen Mythologie, Band 3, Abteilung 2: Pasikrateia-Pyxios, Leipzig.

Scarpi, P. y Ciani, M. G. (1996): Apollodoro, I Miti Greci (Biblioteca), Milán.

Smith, W. (1894): Dictionary of Greek and Roman Biography and Mythology, vol. 3: Oarses-Zygia, Londres.

Usher, M. D. (1998): Homeric Stitchings: The Homeric Centos of the Empress Eudocia, Nueva York.

van Rossum-Steenbeek, M. (1998): Studies on a Selection of Subliterary Papyri, Leiden-Nueva York-Colonia.

Wagner, R. (1891): «Die Sabbaitischen Apollodorfragmente», RhM 46, pp. 378-419. Wagner, R. (1894): Apollodori Bibliotheca, Leipzig. 
Werweyen, T. y Witting, G. (1991): «The Cento. A Form of Intertextuality from Montage to Parody», en Plett, H. F. (ed.), Intertextuality, Berlín-Nueva York, pp. 164-178.

Yun Lee Too (2010): The Idea of the Library in the Ancient World, Oxford.

Fecha de recepción de la primera versión del artículo: 01/06/2020

Fecha de aceptación: 16/11/2020

Fecha de recepción de la versión definitiva: 23/11/2020 